\title{
A Framework for Optimal Control Allocation with Structural Load Constraints
}

\author{
Susan A. Frost ${ }^{1}$ \\ NASA Ames Research Center, Moffett Field, CA, 94035 \\ Brian R. Taylor ${ }^{2}$, Christine V. Jutte ${ }^{3}$, John J. Burken ${ }^{4}$ \\ NASA Dryden Flight Research Center, Edwards, CA, 93523 \\ Khanh V. Trinh ${ }^{5}$ \\ SGT, Moffett Field, CA 94035 \\ and \\ Marc Bodson ${ }^{6}$ \\ University of Utah, Salt Lake City, UT 84112
}

\begin{abstract}
Conventional aircraft generally employ mixing algorithms or lookup tables to determine control surface deflections needed to achieve moments commanded by the flight control system. Control allocation is the problem of converting desired moments into control effector commands. Next generation aircraft may have many multipurpose, redundant control surfaces, adding considerable complexity to the control allocation problem. These issues can be addressed with optimal control allocation. Most optimal control allocation algorithms have control surface position and rate constraints. However, these constraints are insufficient to ensure that the aircraft's structural load limits will not be exceeded by commanded surface deflections. In this paper, a framework is proposed to enable a flight control system with optimal control allocation to incorporate real-time structural load feedback and structural load constraints. A proof of concept simulation that demonstrates the framework in a simulation of a generic transport aircraft is presented.
\end{abstract}

\section{Introduction}

$\mathrm{R}$ educing the environmental impact of civil aviation is a goal of the NASA Aeronautics Subsonic Fixed Wing Project. NASA, industry, universities, and other government organizations are researching advanced technologies and exploring novel civil transport configurations to achieve reductions in noise, emissions, fuel burn, and field length for next generation (NextGen) aircraft ${ }^{1}$. Potential NextGen civil transport aircraft include the Cruise Efficient Short Take-Off and Landing (CESTOL) aircraft and the Hybrid Wing-Body (HWB) aircraft ${ }^{2}$. Some NextGen aircraft configurations are expected to have redundant and multi-purpose control effectors that cannot be easily incorporated into a conventional mixing algorithm that is determined a priori ${ }^{3}$. Additionally, environmental goals may be addressed by using lightweight, flexible composite materials and aircraft configurations with low control authority in some areas of the flight envelope. These considerations suggest optimal control allocation as an approach that could benefit next generation aircraft.

Conventional control allocation schemes control three angular rates in the aircraft body axis primarily with three control variables. Control allocation on NextGen aircraft will control these rates using a variety of redundant and

\footnotetext{
${ }^{1}$ Computer Engineer, Intelligent Systems Division, M/S 269-3, AIAA Member.

${ }^{2}$ Aerospace Engineer, Controls and Dynamics Branch, PO Box 273, M/S 4840D.

${ }^{3}$ Aerospace Engineer, Aerostructures Branch, PO Box 273, M/S 4820-2A, ASME Member.

${ }^{4}$ Aerospace Engineer, Controls Branch, PO Box 273, M/S 4840D, AIAA Senior Member.

${ }^{5}$ Research Scientist, NASA Ames Research Center, M/S 269-3.

${ }^{6}$ Professor, Electrical and Computer Engineering, 50 S Central Campus Dr. Rm. 3280, AIAA Senior Member.
} 
multi-objective control surfaces. We say a vehicle is over-actuated if it has more control effectors than control variables. The control allocation of over-actuated vehicles has been formulated as a constrained optimization problem by many researchers ${ }^{4-14}$. A real-time solution to the control allocation problem is desirable to enable the system to run on NextGen aircraft during flight. This has prompted the search for numerical optimization methods that have good convergence properties and acceptable computational requirements.

Several methods to solve the control allocation problem have been evaluated, including direct allocation, linear programming, quadratic programming, weighted pseudo-inverse, cascaded generalized inverse, and mixed optimization approaches $^{4-14}$. There are advantages and disadvantages to all of the approaches. Control allocation research has also extended the control solution to include coupling or interaction effects between control effectors, creating a nonlinear optimization problem that can often be transformed into a sequence of linear problems ${ }^{14}$. While the interaction effects will be important to study for control allocation in next generation aircraft, this paper will focus on solutions that assume a linear relationship between the effectors and the moments they generate.

While optimal control allocation in flight control systems of fixed wing aircraft is now feasible, given the increased computational resources available on the aircraft, few studies have addressed the structural loads generated on the aircraft due to commanded control surface deflections. For real-time optimal control allocation to be deployed on a civil aircraft, there must be guarantees that the structural load limits of the aircraft will not be exceeded during flight. In Ref. 15, a new mixing law for a flexible transport aircraft was proposed to alleviate the wing bending moment during a sudden and strong roll maneuver with the additional goal of preserving nominal flight behavior. While the approach used an optimization algorithm to choose the control surface commands to be used for maneuver load alleviation, it was an off-line solution that was not used for real-time control allocation during normal flight.

In this paper, a framework is proposed to enable a flight control system with optimal control allocation to incorporate real-time structural load feedback and structural load constraints and ensure the aircraft's structural load limits are not exceeded. A structural model is coupled with a simulation of a generic transport aircraft. The coupled model is used to estimate structural loads on the aircraft during flight and to predict loads generated by control surface deflections. The simulation is used to demonstrate and evaluate the proposed framework, which makes use of structural load feedback, structural load modeling, and optimal control allocation.

\section{Optimization Formulations of Control Allocation}

\section{A. Control allocation in model reference control}

We introduce control allocation in the context of model reference control (a form of dynamic inversion). However, solutions may be used in a variety of control design methods. To state the problem mathematically, we consider the state-space model

$$
\begin{aligned}
& \dot{x}_{A}=A_{A} x_{A}+B u+d \\
& y_{A}=C x_{A}
\end{aligned}
$$

where $x_{A} \in \mathrm{R}^{\mathrm{n}}, d \in \mathrm{R}^{\mathrm{n}}, u \in \mathrm{R}^{\mathrm{p}}, y_{A} \in \mathrm{R}^{\mathrm{q}}$. For the control of aircraft, the states are given by the vector $x_{A}$ and may include the angle of attack, the pitch rate, the angle of sideslip, the roll rate, and the yaw rate $(n=5)$. The output vector $y_{A}$ may contain the pitch rate, the roll rate, and the yaw rate $(q=3)$. The control input vector $u$ consists of the commanded actuator positions. In a conventional aircraft, these commands are the deflections of two elevators, two ailerons, and the rudder $(p=5)$. The disturbance vector $d$ represents the forces and moments that the control surfaces must cancel in order to trim the aircraft (i.e., to create an equilibrium of the dynamical system).

For the purpose of example, consider a simple model reference control law. The method relies on a reference model that represents the desired dynamics of the closed-loop system

$$
\dot{y}_{M}=A_{M} y_{M}+B_{M} r_{M}
$$

where $r_{M}$ is a reference input vector (the pilot commands) and $y_{M}$ represents the desired output of the system. Since the derivative of $y$ is given by

$$
\dot{y}_{A}=C A_{A} x_{A}+C B u+C d
$$


the objective may be achieved by setting

$$
C B u=-C A_{A} x_{A}-C d+A_{M} y_{A}+B_{M} r_{M} \triangleq a_{d}
$$

where $a_{d}$ represents the desired vector to be matched by $C B u$. If $y$ is a vector composed of the incremental rotational rates (as is typically the case), $a_{d}$ represents the desired incremental rotational accelerations, and $u$ represents the incremental surface deflections.

Obtaining $u$ from $a_{d}$ requires that one solve a system of linear equations with more unknowns than equations. Solving such a system is easy, but the difficulty in control allocation is that the vector $u$ is constrained. The limits generally have the form

$$
u_{\min , i} \leq u_{i} \leq u_{\max , i} \quad \text { for } i=1, \ldots, p
$$

where $p$ is the number of surfaces. In vector form, Eq. 5 is written as $u_{\min } \leq u \leq u_{\max }$. There may be additional constraints due to the maximum rate of deflection of the actuators. We refer to the problem of finding a vector $u$ that is the "best" possible solution of Eq. 4 within the constraints Eq. 5 as the control allocation problem.

Given the constraints, the control allocation problem may be such that:

- many solutions exist,

- only one solution exists,

- no exact solution exists.

One is naturally drawn to finding solutions that minimize the error $C B u-a_{d}$. Indeed, providing all the control authority available may make the difference between a maneuver being achievable or not, and between an unusual condition being recoverable from or not. However, the question also arises as to which solution is the most desirable when many solutions exist. Therefore, optimal control allocation typically consists both of error minimization and control optimization. As we will discuss in this paper, the objective of load minimization, or at least load limiting, may also become part of the control allocation problem.

\section{B. Formulations of optimal control allocation}

The fundamental control allocation problem can be formulated as the following error minimization objective.

Error minimization: given a matrix $C B$, find a vector $u$ such that

$$
J=\left\|C B u-a_{d}\right\|
$$

is minimized, subject to $u_{\min } \leq u \leq u_{\max }$.

The problem is solved exactly if $J=0$. However, regardless of whether an exact solution exists, the following control minimization problem may be considered as well.

Control minimization: given a matrix $C B$, a vector $u_{p}$, and a vector $u_{1}$ such that $u_{\min } \leq u_{1} \leq u_{\max }$, find a vector $u$ such that

$$
J=\left\|u-u_{p}\right\|
$$

is minimized, subject to

$$
(C B) u=(C B) u_{1}
$$

and $u_{\min } \leq u \leq u_{\max }$.

The control minimization problem is a secondary optimization objective to be satisfied if the solution of the primary objective, given by $u_{l}$, not unique. The vector $u_{p}$ represents some preferred position of the actuators (e.g., one that yields zero deflections of the surfaces). After a solution yielding minimum error is obtained, the solution with minimum deviation from the preferred position is picked among all equivalent solutions. For both problems, weighting of the elements of the vectors may be inserted in the norms, either to prioritize the axes or to prioritize the actuators. 
The norm used in the optimization criteria is a design choice that has more consequences than might be expected. The $l_{l}$ norm of a vector $x$ is the sum of the absolute values of the elements of the vector

$$
\|x\|_{1}=\sum_{i=1}^{n}\left|x_{i}\right|
$$

while the $l_{2}$ norm is the usual Euclidean norm

$$
\|x\|_{2}=\sqrt{\sum_{i=1}^{n}\left|x_{i}\right|^{2}}
$$

and the $l_{\infty}$ norm is the sup norm

$$
\|x\|_{\infty}=\underset{i}{\max }\left|x_{i}\right|
$$

Algorithms have been proposed for all three norms and the results of the optimization problems are sometimes quite different ${ }^{17,18}$.

A possible implementation of optimization for control allocation consists in the sequential minimization of the error vector and of the control vector. Specifically, the error is minimized first, and then the control vector is minimized among all equivalent solutions. In Ref. 8, the control minimization problem was solved only when the solution of the primary error minimization problem was $J=0$. However, it should be noted that, unless the matrix $C B$ satisfies specific conditions (any $q \times q$ submatrix of $C B$ must be nonsingular), the solution is not necessarily unique, even if the desired vector $a_{d}$ is not feasible. Given this fact, mixed optimization makes sense, and has several advantages over sequential optimization.

Mixed optimization: Given a matrix $C B$ and a vector $u_{p}$, find a vector $u$ such that

$$
J=\left\|C B u-a_{d}\right\|+\varepsilon\left\|u-u_{p}\right\|
$$

is minimized, subject to $u_{\min } \leq u \leq u_{\max }$.

The mixed optimization problem combines the error and control minimization problems into a single problem through the use of a small parameter $\varepsilon$. If the parameter $\varepsilon$ is small, priority is given to error minimization over control minimization, as is normally desired. Often, the combined problem may be solved faster, and with better numerical properties, than when the error and control minimization problems are solved sequentially ${ }^{4}$.

In Ref. 16, an algorithm is proposed that uses the $l_{\infty}$ norm on the control minimization part of the optimization problem. This min-max criterion results in a type of resource balancing, where the resources are the control surface deflections and the algorithm balances those resources to achieve the desired command. Numerical examples demonstrated that this algorithm did a better job of balancing the control surface resources than algorithms that used the $l_{l}$ norm. Advantages of the resource-balancing feature were shown to include a greater resilience to actuator failures and to nonlinear effectiveness for large actuator deflections. An algorithm that computes the $l_{\infty}$ norm of the actuator deflections scaled by the actuator limits, a sort of normalized $l_{\infty}$ norm, results in even better resource balancing ${ }^{18}$. Algorithms that use the $l_{\infty}$ norm on the control effort with scaling translate into minimization of the maximum actuator deflection as a percentage of its range of motion.

Computational studies have shown that the solution of control allocation methods can be sensitive to the value of the desired acceleration vector. A high sensitivity may yield to actuator rate saturation in the case of rapid changes of command. Reducing the sensitivity lowers the risk that rate limits will be encountered. Studies of the effect of the norm on the control effort in the mixed optimization problem have shown that an algorithm formulated with the $l_{l}$ norm on the control cost has higher sensitivity than an algorithm formulated with the $l_{2}$ or the $l_{\infty}$ norm, while an

algorithm using the normalized $l_{\infty}$ norm has the lowest sensitivity ${ }^{17,18}$. However, the solution of the problem with normalized $l_{\infty}$ norm was also the most computationally demanding.

\section{Implementation of optimal control allocation algorithms}


Computational resources available on modern aircraft make the use of optimal control allocation algorithms feasible in real-time. An efficient algorithm to solve the mixed optimization problem given in Eq. 12 with the $l_{l}$ norm on the criterion was formulated by Bodson using linear programming approaches, providing guaranteed convergence to a solution in an acceptable period of time ${ }^{4}$. Timing data showed that solutions of the problem could comfortably be performed in real-time, even for large numbers of actuators, and that the optimal solution improved performance significantly over simpler, approximate methods ${ }^{11}$. The algorithm was based on the revised simplex method ${ }^{19}$ with additional refinements, such as anticycling, as described in detail in Ref. 4.

Harkegård proposed an elegant solution of the optimal control allocation problem using the $l_{2}$ norm and the theory of active sets ${ }^{12}$. The algorithm was very similar to the simplex algorithm used for $l_{l}$ optimization, and had the same advantage of completing in finite time and with a small number of iterations.

In Ref. 16, the $l_{l}$ norm is used for the error minimization and the $l_{\infty}$ norm is used for control minimization, with both criteria combined in a single, mixed optimization criterion. A small modification of the approach used for mixed $l_{l}$ optimization yields the desired linear program. A further modification to the algorithm using the $l_{\infty}$ norm for control minimization yields the solution of a new problem where the actuator deflections are weighted in the computation of the $l_{\infty}$ norm as per unit values, where a unit is the maximum deflection of the actuator ${ }^{18}$. In this algorithm, minimization of the control effort translates into minimization of the maximum actuator deflection as a percentage of its range of motion.

\section{Control Allocation with Structural Load Constraints and Load Feedback}

Most optimal control allocation algorithms find an optimal solution to the control allocation problem within the constraints of the control surface position and possibly rate limits. However, these constraints are not sufficient to ensure that the structural load limits of the aircraft will not be exceeded by the commanded control surface deflections. The bending and torsion moments at the wing root are examples of loads on the aircraft that need to be monitored. Structural load constraints will need to be accounted for in order to deploy optimal control allocation on NextGen aircraft. In this section, we formulate the load constraints at discrete critical points on the aircraft as

$$
\|M+T u\| \leq L_{\max }
$$

where $\boldsymbol{M}$ is a vector of the current measured or estimated loads at the critical points, $\boldsymbol{T}$ is a matrix that converts the effect of incremental surface deflections into incremental structural loads, and $\boldsymbol{L}_{\max }$ is a vector of maximum allowable structural loads at the critical points. The loads that need to be limited are a function of the aircraft being considered, often with an emphasis on torsion and bending moments. Generally, the load limits are determined through detailed studies, including ground and flight tests. This paper will not address the selection of the location or the number of load points to be considered for a given problem. For the purpose of developing a representative example, we choose load points along the aircraft wing.

We assume that the $\boldsymbol{T}$ matrix, which is computed from the states of the aircraft at the current time, gives a linear approximation of the incremental structural loads arising from commanded surface deflections. The incremental loads matrix is formed by perturbing each control surface deflection from its current position at the current aircraft state. The perturbation yields the change in aerodynamic lift and rolling moments due to a one-degree change in surface deflection. It is assumed that the entire aircraft lift is distributed elliptically across the wing. It is also assumed that the control effectiveness of each surface is proportional to the lift generated by that control surface. The resulting lift and moment components are used in conjunction with a structural model of the aircraft to determine moments at critical points on the aircraft. Superposition of the control surface effects in terms of lift, moments, and structural loads is assumed in order to obtain a reasonable, but tractable solution in real-time.

The structural load limits can be implemented as an additional constraint as given in Eq. 13. Additionally, the control allocation cost function can include a term to minimize the loads at the critical points, as given below

$$
J=\left\|C B u-a_{d}\right\|+\varepsilon\left\|u-u_{p}\right\|+\gamma\|M+T u\|
$$

The $l_{\infty}$ norm could be used on the load minimization criterion in Eq. 14 in an implementation that is similar to its use on the control minimization described in Section II (c). The critical load points could be weighted in the computation of the $l_{\infty}$ norm as per unit values, where a unit is the maximum load limit of the critical point. Using this approach, solutions could be obtained that more evenly distribute loads at the critical points. 


\section{Proof of Concept Simulation Architecture}

In this section we describe the proof of concept simulation implemented to demonstrate the proposed framework assuming static loads, an elliptical lift distribution, and a limited number of critical load points.

\section{A. Aircraft configuration and aerodynamic model}

A full nonlinear simulation of a class of full-scale generic transport aircraft is used for the proof of concept study of the proposed framework. The simulation uses a dynamically scaled representative transport model that is derived from a model of NASA's AirSTAR testbed. The AirSTAR testbed is being developed as part of NASA's Aviation Safety Program to investigate dynamics modeling and control of large transport vehicles in upset conditions ${ }^{20}$. The AirSTAR unmanned aerial vehicle (UAV) is a 5.5\% dynamically scaled aircraft based on wind tunnel and flight test data $^{21}$. The simulation used in this paper is a full-scale model that was derived from the subscale model by incorporating Reynolds adjusted aero tables, actuator models appropriate for a full-scale aircraft, and a model of NASA Glenn's CMAPSS “simp2” simplified engine models.

The simulation created for this study represents a conventional modern midsize commercial passenger configuration. The aircraft has right and left inboard and outboard elevons, three ailerons on each wing, and upper and lower rudders for use by the control allocator to achieve the desired roll, pitch, and yaw moments commanded by the flight control system. A stabilizer is used for trimming the aircraft.

For the proof of concept study, eight critical points on the aircraft were monitored for bending moments. These critical points were located on each wing at the wing root and at an inboard location on each of the three ailerons. The outboard ailerons and the corresponding critical points are labeled AILL3 and AILR3, respectively, for the left and right wings. Comparisons will be made between the allocator having no load limits and having load limits. When load limits are used by the allocator, they are set to $6700 \mathrm{ft}-\mathrm{lb}$ bending moments at the outboard ailerons, AILL3 and AILR3, and $5 \times 10^{6} \mathrm{ft}-\mathrm{lb}$ bending moments for the other critical points. The outboard aileron limits are set below the loads recorded during unconstrained flight as seen in the results section below. These constraints were chosen to demonstrate the ability of the control allocator to remain within structural load limits, while achieving the desired commands. Further analysis will be done to define more realistic load limits.

\section{B. Static structural model}

A finite element analysis (FEA) model is developed and integrated into the simulation to estimate static structural loads on the aircraft due to lift and roll moments and to compute the incremental loads created by surface deflections. For the proof of concept study, only bending moments at the wing-root and aileron locations are monitored. The finite element method is employed for its ability to calculate wingspan internal loads for potentially complex wingspan load distributions and wing geometries. The basic FEA model requires a small number of matrix multiplications for static loads analysis. Furthermore, the finite element method provides an efficient framework for adding additional monitored points. In implementing this approach, we hope to determine whether the required computations are fast enough and accurate enough for flight controllers in both real-time simulations and actual flight.

The FEA modeling is simplified to beam modeling, based on mass and stiffness approximations for a representative full-scale generic civil transport model. The model includes two beam meshes for representing the left and right wings independently. Figure 1 shows the two wing meshes with the node numbers. Each wing mesh has 20 nodes and 19 beams. The wings are modeled as cantilever beams with the fixed ends at the wing roots. All degrees of freedom for node 1 are fixed for both meshes. The global coordinates of the finite element model are attached to the aircraft, with the origin at the nose, positive $X$-axis pointing toward the tail, and positive $Z$-axis pointing up. We use a simplified approximation of the wing as hollow aluminum shells having the wing outer mold line of the representative aircraft and a constant thickness of five inches. Beam cross section properties are calculated using this hollow shell wing geometry. Figure 2 shows nodes of the right wing beam mesh running through centroids of the wing cross sections.

The $\mathrm{C}^{0}$ approach as described in Ref. 23 is used for the beam modeling. There are three translation and three rotation degrees of freedom per node. A linear shape function with one-point reduced integration is used for the axial, transverse shear, torsion and transverse bending components. The "residual bending flexibility" technique ${ }^{23}$ is also applied to the shear term to improve the accuracy of the two-node, one-point quadrature beam element. The displacement solution for the static problem is given by

$$
d=K^{-1} F
$$


where $\boldsymbol{d}$ is a vector containing the displacement solutions, $\boldsymbol{K}$ is the stiffness matrix of the wing structure, and $\boldsymbol{F}$ is a vector of external loads applied to the structure. All terms in Eq. 15 are calculated in the global coordinate system. The internal moment about local beam direction $2, \boldsymbol{M}_{2}$, is the bending moment used as the measured load at the critical points by the control allocator in the simulation. The calculation for the internal moment for the beams is given by

$$
M_{2}=E I_{22} k_{22}
$$

where $\boldsymbol{E}$ is Young's modulus, $\boldsymbol{I}_{22}$ is the second moment of inertia about the local 2 beam axis, and $\boldsymbol{k}_{22}$ is the curvature about the local 2 beam axis, which is calculated from the displacement solutions and the beam finite element shape function.

The structural modeling and analysis used for this study assumes static conditions and considers external loads on the wing due to aerodynamic lift forces and roll moments. The lift distribution on each wing is assumed to be elliptical, with each wing carrying half of the total aircraft lift. Figure 3 shows the normalized lift per length distribution for each wing. The external loads arising from the roll moment on the aircraft wing are assumed to be from concentrated forces applied in the aircraft $Z$ direction at the ailerons. These forces are applied to nodes 15,17 , and 19 , located at the center of the three ailerons on each wing. The $Z$ direction force acting on each aileron is assumed to be proportional to the aileron deflections.

To minimize computation time, $\boldsymbol{K}$ is calculated and inverted in a pre-processing step prior to running the simulation. The normalized lift force of $11 \mathrm{bf}$ with the elliptical distribution shown in fig. 3 is also calculated in the pre-processing step. The total force $\boldsymbol{F}$ on each wing is computed as the sum of (i) the wing's lift using the elliptical lift force distribution and (ii) any roll forces at the aileron nodes $(15,17,19)$. The simulation uses these parameters to compute the displacements as given by Eq. 15. The bending moments at the critical points are then derived from the displacements and the structural model. The aileron critical points are at the aileron inboard edges, nodes 14, 16, and 18. These estimated bending moments are used as the simulated current load measurements at the critical points.

The incremental load matrix $\boldsymbol{T}$ represents the change in internal loads on the wing due to a change in the surface deflection from the current position. It is computed by determining the change in lift and moment forces due to a one-degree change in control surface deflection from the current surface position.

\section{Stability and control augmentation system with optimal control allocation}

The stability and augmentation system for the simulation uses a dynamic inversion controller with a second order reference model. The simulation has multiple surfaces that control multiple axes. The control allocator is able to use these surfaces to achieve the desired roll, pitch, and yaw moments commanded by the flight control system. The allocator has inputs for measured load $\boldsymbol{M}$, the incremental load matrix $\boldsymbol{T}$ for predicting the control surface contribution to the load at the critical points, a preferred position $\boldsymbol{u}_{\boldsymbol{p}}$, surface position limits, and load limits. The load limits are incorporated as a constraint on the cost function. Since this paper is looking at the feasibility of the framework, we are only considering the load constraint on the bending moment at the eight critical points along the wings. The control allocation problem solved in this simulation is to find the vector $u$ such that

$$
J=\left\|C B u-a_{d}\right\|_{1}+\varepsilon\left\|u-u_{p}\right\|_{1}
$$

is minimized, subject to $u_{\min } \leq u \leq u_{\max }$ and $\|M+T u\| \leq L_{\max }$. The above criterion and constraints are converted to a linear program and solved using the revised simplex algorithm described in Section II (c), which was modified to include the load constraints. The control allocator does not have actuator rate limits as a constraint, but the actuators modeled in the simulation rate limit their commands. The control allocator weighted the control minimization criterion by setting $\varepsilon=10^{-3}$. Scaling of some of the inputs to the control allocator was necessary to achieve good numerical properties for the algorithm. The parameters $\boldsymbol{C} \boldsymbol{B}$ and $\boldsymbol{a}_{d}$ were scaled by a factor of $10^{-3}$ and the structural load parameters $\boldsymbol{M}, \boldsymbol{T}$, and $\boldsymbol{L}_{\text {max }}$ were scaled by a factor of $10^{-2}$.

\section{Simulation Results}

Simulations with the architecture described in Section IV were run without load constraints and with load constraints to determine the feasibility of using optimal control allocation with structural load feedback and load constraints. We chose a roll doublet for the proof of concept criterion. The roll doublet can be performed without 
causing excessive maneuver loads that would exceed reasonable load limits. The simulations were run after the aircraft was trimmed at Mach 0.70 and 30,000 feet.

The first simulation describe is run without load limits. Figure 4 shows the commanded rates and the aircraft response to the doublet command. The controller performs adequately for this proof of concept study, although improved tracking is desirable. A feature of optimal control allocation algorithms with $l_{l}$ norms on the control error is that the solutions will be such that the most effective surfaces are used to achieve the command. Since the commanded moments include positive pitch values, the allocator makes use of the elevons, in addition to ailerons, to more effectively achieve roll and pitch. The ailerons that are most effective for roll are the outboard ailerons, AILL3 and AILR3. The aileron deflections for the commanded maneuver are shown in fig. 5. The loads on the right and left outboard aileron critical points are shown in fig.6.

A second simulation is run with bending moment load limits at the outboard ailerons on both wings, AILL3 and AILR3, set to $6700 \mathrm{ft}-1 \mathrm{~b}$, and the other critical point limits set to $5 \times 10^{6} \mathrm{ft}-1 \mathrm{~b}$. Figure 7 shows the commanded rates and the aircraft response to the doublet command. The aircraft's response with load limits is comparable to the response of the simulation without load limits. Since the limits at the outboard ailerons were lower than the bending moments seen in fig. 6, it is clear that the allocator must use different surface commands to achieve the desired moments while remaining within the load constraints. The allocator uses the middle ailerons, AILL2 and AILR2, and some of the inboard aileron to avoid exceeding the load limits, as seen in figs. 8-9. The allocator uses these ailerons since they are able to achieve the roll command without exceeding the load limits at the outboard ailerons. A limitation of this study is that the effects of the roll moments created by the elevons are not considered.

\section{Conclusions and Future Work}

A framework is proposed and demonstrated in simulation, using load constraints and real-time load feedback in conjunction with optimal control allocation. Optimal control allocation was demonstrated to command surface deflections to achieve desired moments while remaining within the defined structural load limits of the aircraft. The runtime of the simulation was reasonable, but it has not been studied for scaling of the problem.

This study reveals many interesting problems for future investigation. It is likely that making use of different norms on the control allocation cost function would provide solutions with more desirable properties. The use of weights on the surfaces and critical load points in the control allocation cost function should also be investigated. Adding a load minimization criterion with the normalized $l_{\infty}$ norm to the control allocation problem will be explored in the hope that this will keep loads at critical points further from their limits.

Robustness of the load model and the incremental loads matrix need to be examined in much more detail. Future work will be done to increase the fidelity of the structural model, add structural dynamics, and include additional load paths. Many challenges will undoubtedly arise when integrating information from the sensed loads on the aircraft with the structural model.

Studies of the integrated flight control system to determine the robustness and sensitivity to errors in the load measurements and incremental loads matrix will be essential before transitioning to a system with load measurements coming from sensors. At this time, the computation time for this simulation is reasonable. However, as the system becomes more complex, methods to increase the computation speed may become necessary. One area of investigation is whether some load paths could be ignored at certain parts of the flight envelope. Another important question is how often the load measurements and the incremental load matrix need and can be updated.

Future work will investigate the extension of the control system framework presented in this paper to gust load alleviation. Sensors, modeling, or a combination of both will provide the current load at critical points while $\boldsymbol{T}$ will give the incremental load per incremental gust. Given an estimate of an upcoming gust, its load can be predicted and an optimal control allocator can use appropriate surfaces to counteract the predicted load.

There are many open areas of research in the use of real-time load feedback in flight control systems. Future aircraft will likely employ some sort of real-time load feedback as materials, sensors, computation power, and aircraft configurations evolve.

\section{Acknowledgments}

Special thanks and recognition for technical guidance given by Diana Acosta of NASA Ames Research Center and Martin Brenner of NASA Dryden Flight Research Center.

\section{References}

\footnotetext{
1“Fundamental Aeronautics Program Subsonic Fixed Wing Project Reference Document," National Aeronautics and Space Administration, May 2006, URL: http://www.aeronautics.nasa.gov/programs fap.htm 
${ }^{2}$ Acosta, D. M., KrishnaKumar, K., and Frost, S. A.. "Towards Intelligent Control for Next Generation Aircraft," Proceedings International Powered Lift Conference, 2008 Royal Aeronautical Society, London, England, July 2008.

${ }^{3}$ Cameron, D., Princen, N., "Control Allocation Challenges and Requirements for the Blended Wing Body," AIAA Guidance, Navigation and Control Conference and Exhibit, AIAA, Denver, Colorado, August 2000, AIAA-2000-4539.

${ }^{4}$ Bodson, M., "Evaluation of Optimization Methods for Control Allocation," AIAA Journal of Guidance, Control, and Dynamics, vol. 25, no. 4., pp.703-711, 2002.

${ }^{5}$ Buffington, J., "Tailless Aircraft Control Allocation," Proc. of the AIAA Guidance, Navigation, and Control Conference, New Orleans, LA, 1997, pp. 737-747.

${ }^{6}$ Durham, W. "Constrained Control Allocation,” Journal of Guidance, Control, and Dynamics, vol. 16, no. 4, 1993, pp. 717725.

${ }^{7}$ Enns, D., "Control Allocation Approaches," AIAA Guidance, Navigation and Control Conference and Exhibit, AIAA, Boston, MA, August 1998, AIAA-1998-4109.

${ }^{8}$ Buffington, J. "Modular Control Law Design for the Innovative Control Effectors (ICE) Tailless Fighter Aircraft Configuration 101-3," Report AFRL-VA-WP-TR-1999-3057, Air Force Research Laboratory, Wright-Patterson AFB OH 45433$7542,1999$.

${ }^{9}$ J. Burken, P. Lu, Z. Wu, \& C. Bahm "Two Reconfigurable Flight-Control Design Methods: Robust Servomechanism and Control Allocation," Journal of Guidance, Control, and Dynamics, vol. 24, no. 3, 2001, pp. 482-493.

${ }^{10}$ J. Petersen \& M. Bodson, "Interior-Point Algorithms for Control Allocation," Journal of Guidance, Control, and Dynamics, vol. 28, no. 3, 2005, pp. 471-480.

${ }^{11}$ J. Petersen \& M. Bodson, "Constrained Quadratic Programming Techniques for Control Allocation," IEEE Trans. on Control Systems Technology, vol. 14, no. 1, January 2006, pp. 91-98.

${ }^{12}$ Härkegård, O., "Efficient Active Set Algorithms for Solving Constrained Least Squares Problems in Aircraft Control Allocation," $41^{\text {st }}$ IEEE Conference on Decision and Control, Vol. 2, pp. 1295-1300.

${ }^{13}$ Page, A. B., Steinberg, M. L., "High-Fidelity Simulation Testing of Control Allocation Methods," AIAA Guidance, Navigation and Control Conference and Exhibit, AIAA, Monterey, California, August 2002, AIAA-2002-4547.

${ }^{14}$ Oppenheimer, M. W. and Doman, D. B., "A Method for Including Control Effector Interactions in the Control Allocation Problem," report AFRL-VA-WP-TP-2007-309, Air Force Research Laboratory, Wright-Patterson AFB, OH 45433-7542, 2007.

${ }^{15}$ Gaulocher, S.L., Roos, C., and Cumer, C., "Aircraft Load Alleviation during Maneuvers Using Optimal Control Surface Combinations," Journal of Guidance, Control, and Dynamics, vol. 30, no. 2, March 2007, pp. 591-600.

${ }^{16}$ Bodson, M. \& Frost, S., "Control Allocation with Load Balancing," Proceedings of the AIAA Guidance, Navigation and Control Conference and Exhibit, AIAA, Chicago, IL, August 2008, AIAA.

${ }^{17}$ Frost, S.A., Bodson, M., \& Acosta, D.M., "Sensitivity Analysis of Linear Programming and Quadratic Programming Algorithms for Control Allocation," AIAA InfoTech@Aerospace Conference, Seattle, WA, 2008, AIAA.

${ }^{18}$ Frost, S.A. \& Bodson, M, "Resource Balancing Control Allocation," American Control Conference, Baltimore, MD, 2010.

${ }^{19}$ Luenberger, D., Introduction to Linear and Nonlinear Programming, Addison-Wesley, Reading, MA 1984, pp. 80.

${ }^{20}$ Jordan, T.L., Foster, J.V., Bailey, R.M., \& Belcastro, C.M., "AirSTAR: A UAV Platform for Flight Dynamics and Control System Testing", AIAA 2006-3307, 25th AIAA Aerodynamic Measurement Technology and Ground Testing Conference, AIAA, San Francisco, CA, 2006.

${ }^{21}$ Jordan, T.L., Langford, W.M., and Hill, J.S., "Airborne Subscale Transport Aircraft Research Testbed, Aircraft Model Development”, AIAA 2005-6432, AIAA Guidance, Navigation, and Control Conference, AIAA, Washington, DC, 2005.

22"Quadratic Programming Control Allocation Toolbox (QCAT)," Matlab® Central, August 2008, URL: http://www.mathworks.com/matlabcentral/fileexchange/loadFile.do?objectId=4609\&objectType=file

${ }^{23}$ Hughes, Thomas J.R., The Finite Element Method-Linear Static and Dynamic Finite Element Analysis, Springer-Verlag, New York, 1983, Chapter 5. 


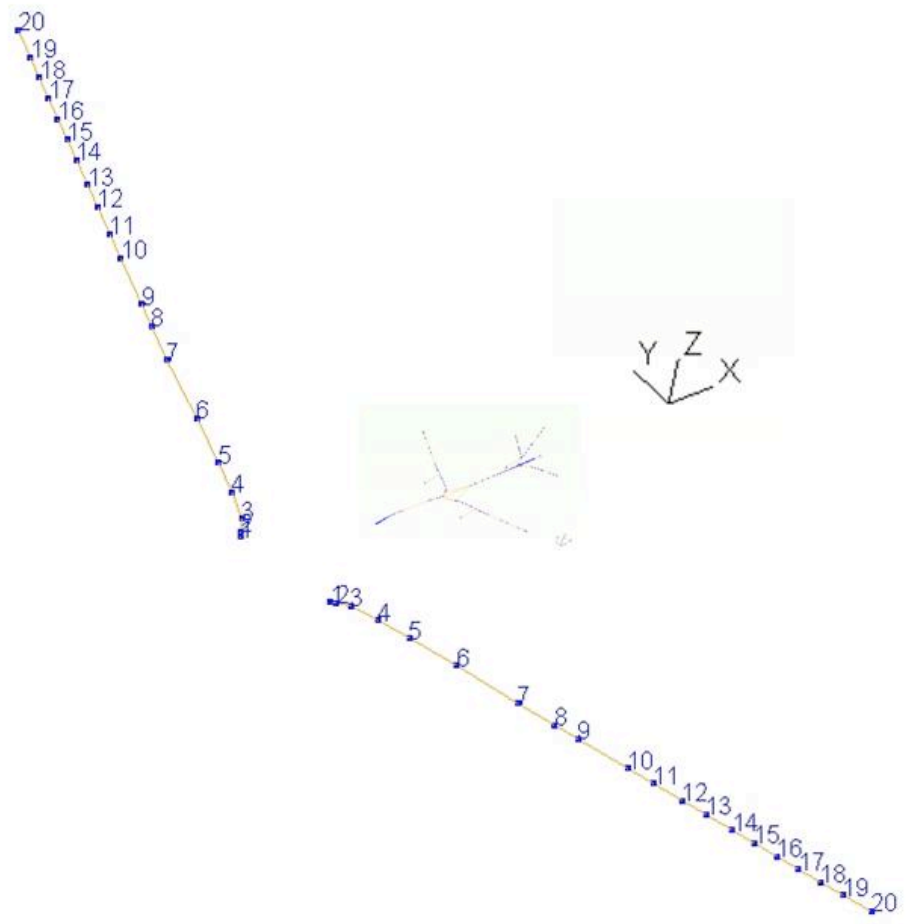

Fig. 1. Finite element beam model for both left and right wing.

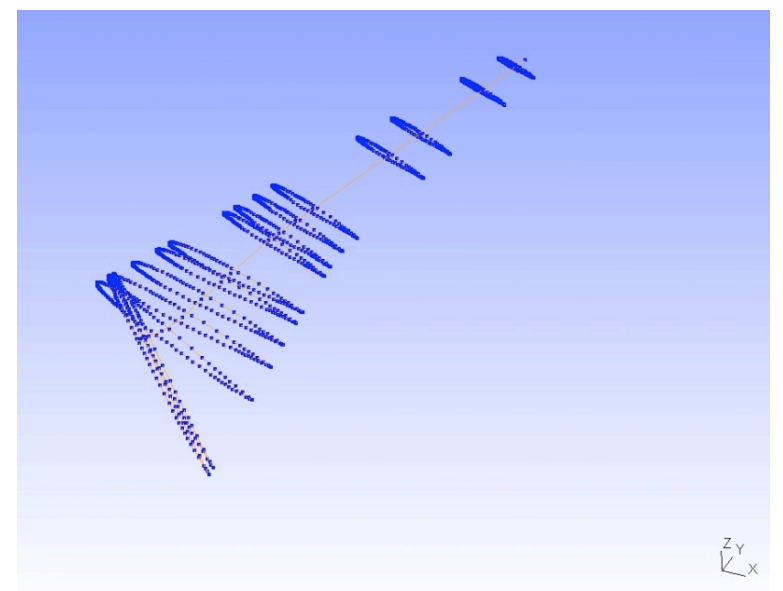

Fig. 2. Beam nodes located at centroids of wing cross sections. 


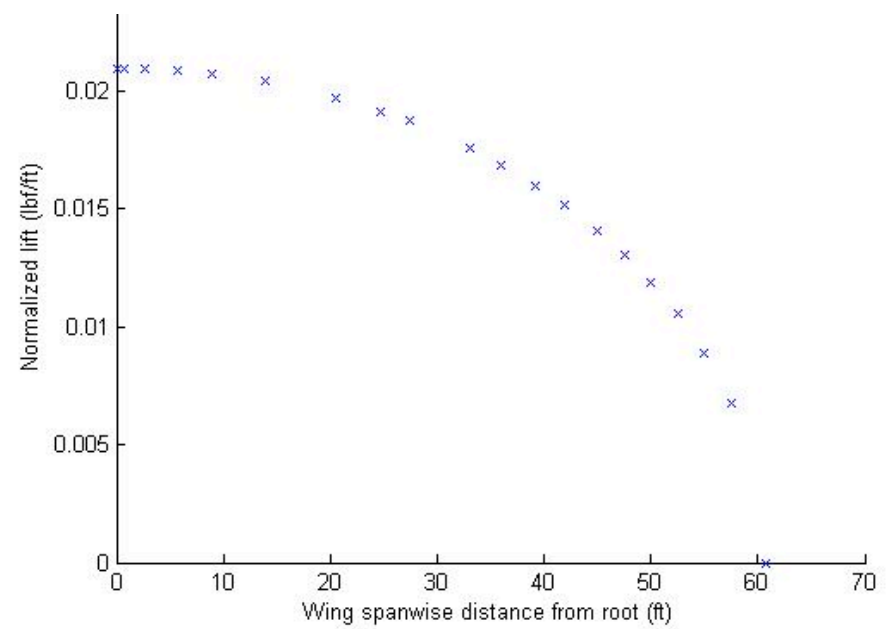

Fig. 3. Normalized lift load on each wing (lbf/ft).
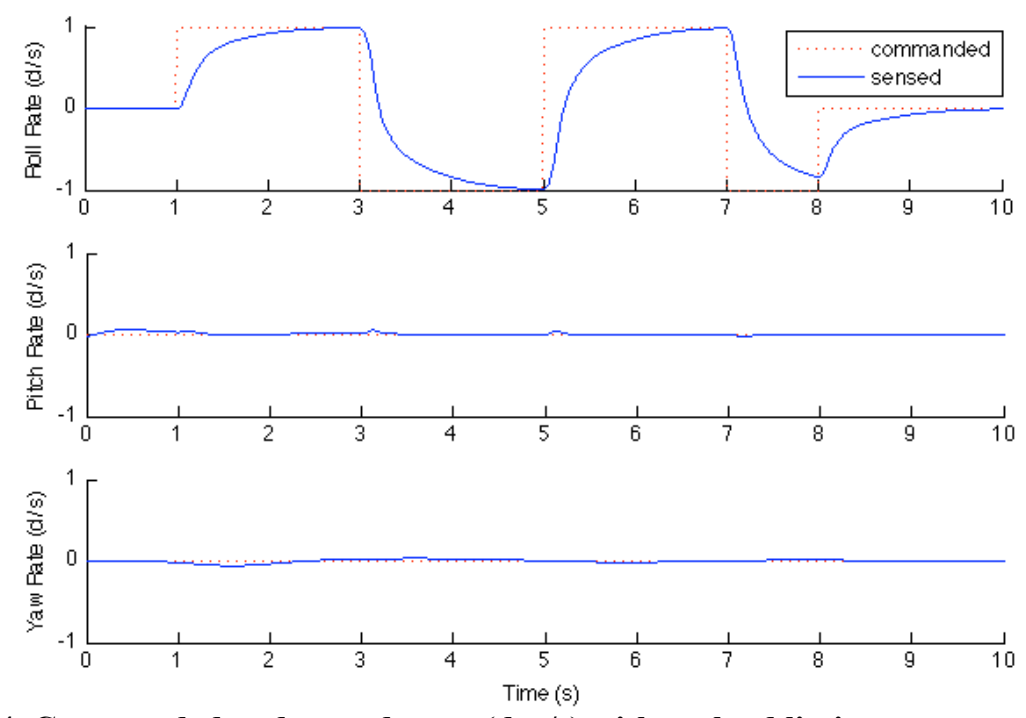

Fig. 4. Commanded and sensed rates (deg/s) without load limits.
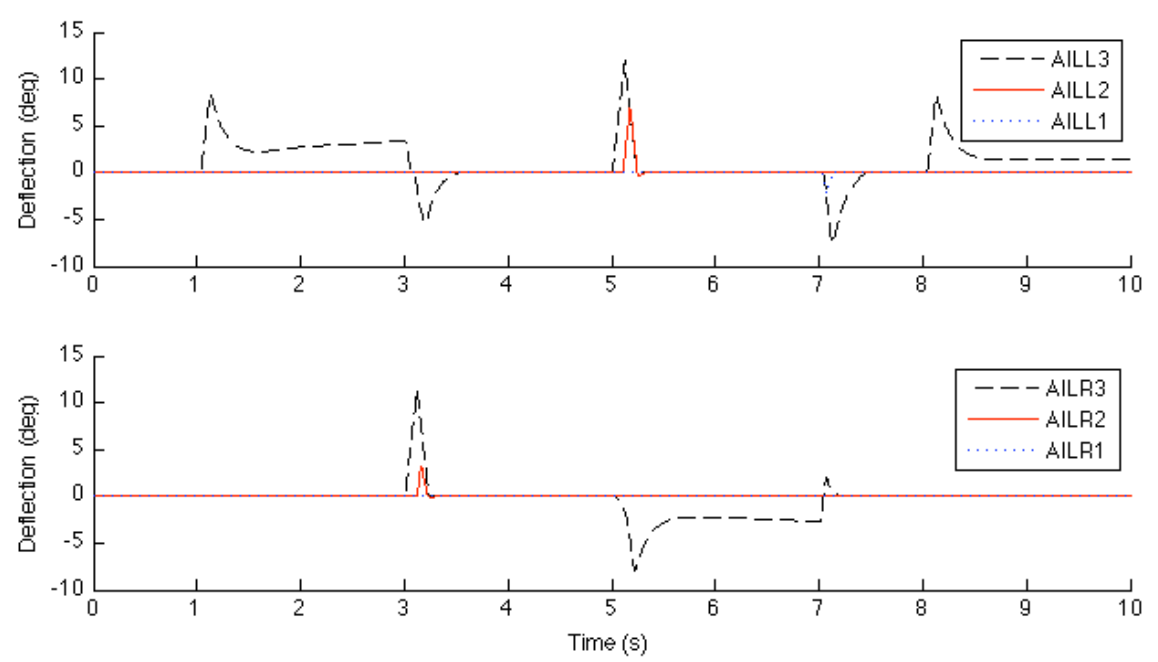

Fig. 5. Aileron deflections (deg) on left wing (top) and right wing (bottom) without load limits. 

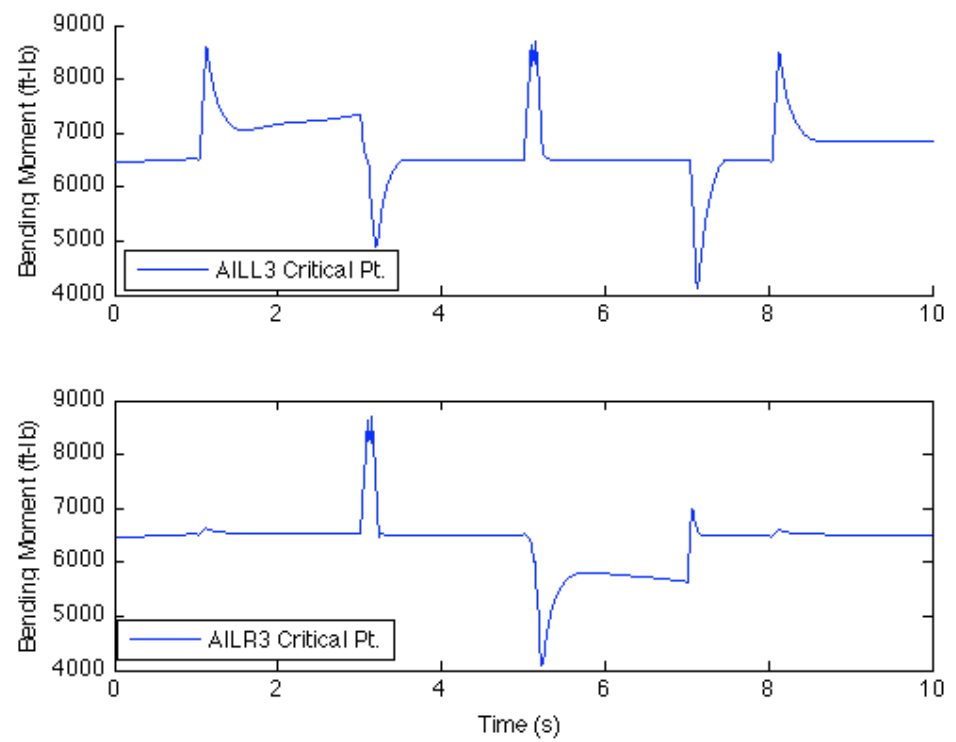

Fig. 6. Bending moment (ft-lb) at left and right aileron 3 critical points without load limits.
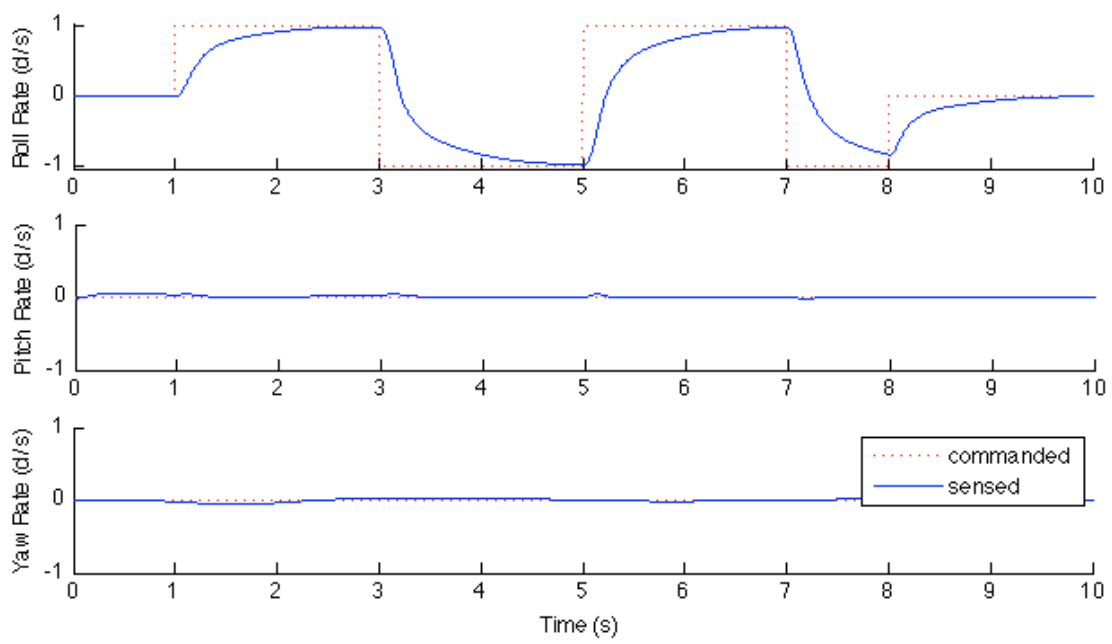

Fig. 7. Commanded and sensed rates (deg/s) with load limits.
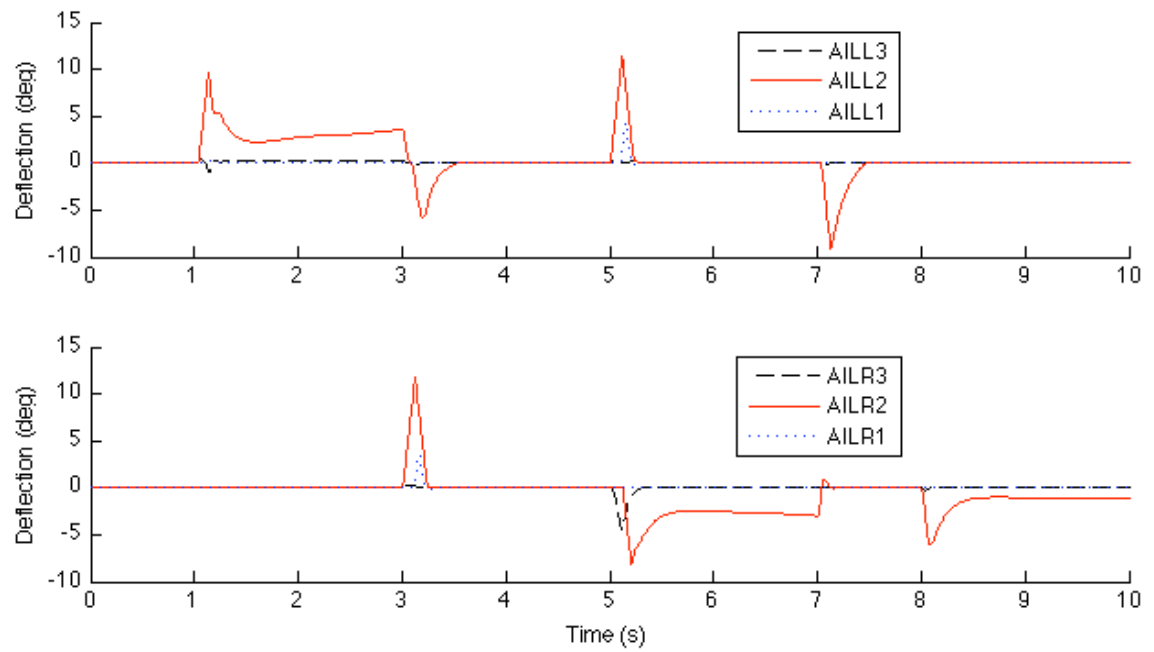

Fig. 8. Aileron deflections (deg) on left wing (top) and right wing (bottom) with load limits.

12

American Institute of Aeronautics and Astronautics 

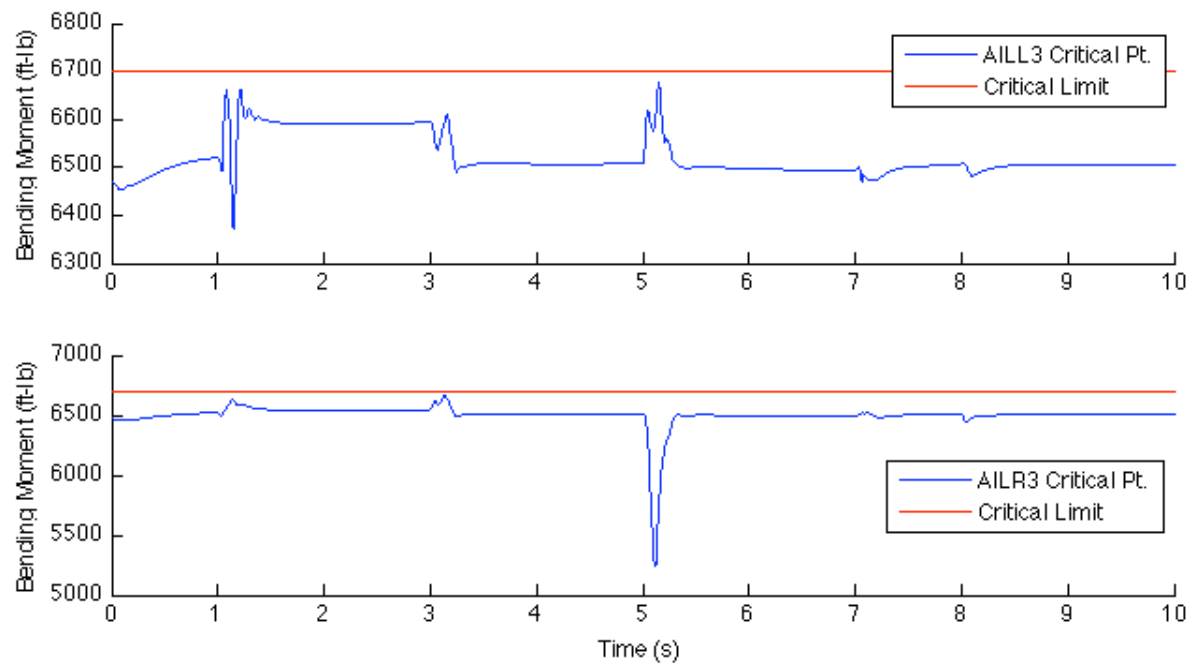

Fig. 9. Bending moment (ft-lb) at left (top) and right (bottom) aileron 3 critical points with load limits. 